\title{
Density functional theory investigation of benzenethiol adsorption on $\mathrm{Au}(111)$
}

\author{
Nara, Jun; Higai, Shin’ichi; Morikawa, Yoshitada; Ohno, Takahisa
}

Published in:

Journal of Chemical Physics

Link to article, DOI:

$10.1063 / 1.1651064$

Publication date:

2004

Document Version

Publisher's PDF, also known as Version of record

Link back to DTU Orbit

Citation (APA):

Nara, J., Higai, S., Morikawa, Y., \& Ohno, T. (2004). Density functional theory investigation of benzenethiol adsorption on Au(111). Journal of Chemical Physics, 120(14), 6705-6711. https://doi.org/10.1063/1.1651064

\section{General rights}

Copyright and moral rights for the publications made accessible in the public portal are retained by the authors and/or other copyright owners and it is a condition of accessing publications that users recognise and abide by the legal requirements associated with these rights.

- Users may download and print one copy of any publication from the public portal for the purpose of private study or research.

- You may not further distribute the material or use it for any profit-making activity or commercial gain

- You may freely distribute the URL identifying the publication in the public portal

If you believe that this document breaches copyright please contact us providing details, and we will remove access to the work immediately and investigate your claim. 


\title{
Density functional theory investigation of benzenethiol adsorption on Au(111)
}

\author{
Jun Nara ${ }^{a)}$ and Shin'ichi Higai \\ National Institute for Materials Science (NIMS), 1-2-1 Sengen, Tsukuba-shi, Ibaraki 305-0047, Japan \\ Yoshitada Morikawa \\ Research Institute for Computational Sciences (RICS), and Research Consortium for Synthetic Nano-Function \\ Materials Project (SYNAF), National Institute of Advanced Industrial Science and Technology (AIST), \\ Tsukuba Central 2, 1-1-1 Umezono, Tsukuba-shi, Ibaraki 305-8568, Japan \\ and Center for Atomic-scale Materials Physics, Department of Physics, Technical University of Denmark, \\ DK-2800 Lyngby, Denmark \\ Takahisa Ohno \\ National Institute for Materials Science (NIMS), 1-2-1 Sengen, Tsukuba-shi, Ibaraki 305-0047, Japan \\ and Institute of Industrial Science, University of Tokyo, 4-6-1 Komaba, Meguro-ku, Tokyo 153-8505, Japan
}

(Received 2 September 2003; accepted 7 January 2004)

\begin{abstract}
We have studied the adsorption of benzenethiol molecules on the $\mathrm{Au}(111)$ surface by using first principles total energy calculations. A single thiolate molecule is adsorbed at the bridge site slightly shifted toward the fcc-hollow site, and is tilted by $61^{\circ}$ from the surface normal. As for the self-assembled monolayer $(\mathrm{SAM})$ structures, the $(2 \sqrt{3} \times \sqrt{3}) R 30^{\circ}$ herringbone structure is stabilized against the $(\sqrt{3} \times \sqrt{3}) R 30^{\circ}$ structure by large steric relaxation. In the most stable $(2 \sqrt{3} \times \sqrt{3}) R 30^{\circ}$ SAM structure, the molecule is adsorbed at the bridge site with the tilting angle of $21^{\circ}$, which is much smaller compared with the single molecule adsorption. The van der Waals interaction plays an important role in forming the SAM structure. The adsorption of benzenethiolates induces the repulsive interaction between surface $\mathrm{Au}$ atoms, which facilitates the formation of surface $\mathrm{Au}$ vacancy. () 2004 American Institute of Physics. [DOI: 10.1063/1.1651064]
\end{abstract}

\section{INTRODUCTION}

Self-assembled monolayers (SAMs) of organic molecules on metal surfaces are promising systems to open up a door to exotic physical phenomena as well as new technologies, such as in surface coating, wetting, chemical sensing, and other various surface functionalizations. The SAMs of alkanethiol molecules on gold surfaces are the prototypical systems because of the simplicity of the molecules, their high stability, and the ease-to-preparation, and have been extensively investigated in the past decades. ${ }^{1-3}$ Aromatic thiols, on the other hand, have recently come to draw much attention due to their potential applications, especially in molecular electronic devices, taking advantage of their high conductivity and nonlinear optical properties. ${ }^{4,5}$ For example, the charge transport through single molecules has been reported for the system of benzene-(1,4)-dithiols sandwiched with two gold electrodes. ${ }^{5}$ Geometric information for organic molecules adsorbed on metal surfaces is essential for understanding the electronic and optical properties of materials and devices based on these systems. Much less is, however, known about the aromatic thiol systems compared with the alkanethiol ones.

Benzenethiol $\left(\mathrm{C}_{6} \mathrm{H}_{5} \mathrm{SH}\right)$ is one of the simplest aromatic thiols. The adsorption of benzenethiol on metal surfaces has been experimentally investigated in recent years. The benzenethiol is known to be dissociatively adsorbed on metal

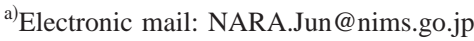

surfaces via the $\mathrm{S}$ atom to form benzenethiolate $\left(\mathrm{C}_{6} \mathrm{H}_{5} \mathrm{~S}\right)$, and the benzenethiolate is tilted from the surface normal. ${ }^{6,7}$ Although there are some cluster calculations ${ }^{8,9}$ reported besides experiments, reliable information on the orientation of the benzenethiolate as well as the adsorption site of the $\mathrm{S}$ atom is still not available. As for the surface ordering of SAM structures, Dhirani et al. reported that oligo(phenylethynyl) benzenethiols form well-ordered SAM phases on the $\mathrm{Au}(111)$ surface with $(2 \sqrt{3} \times \sqrt{3})$ symmetry whereas the benzenethiol does not form an ordered SAM. ${ }^{10}$ Wan et al., on the other hand, reported that the benzenethiol forms a well-ordered monolayer. ${ }^{11}$ It is also still a controversial issue whether the benzenethiol forms any ordered phase on the $\mathrm{Au}(111)$ surface.

In this paper, we investigate the adsorption geometry of benzenethiolates on the $\mathrm{Au}(111)$ surface both in low coverage and in SAM structures, by using ab initio total energy calculation techniques.

\section{COMPUTATIONAL DETAILS}

We have performed first-principles total-energy calculations based on the density functional theory (DFT) by using a program package STATE, which has been successfully applied for alkanethiol self-assembled monolayers on $\mathrm{Au}(111) .{ }^{12-14}$ The spin polarized generalized gradient approximation (GGA) in Perdew-Burke-Ernzerhof formula is 


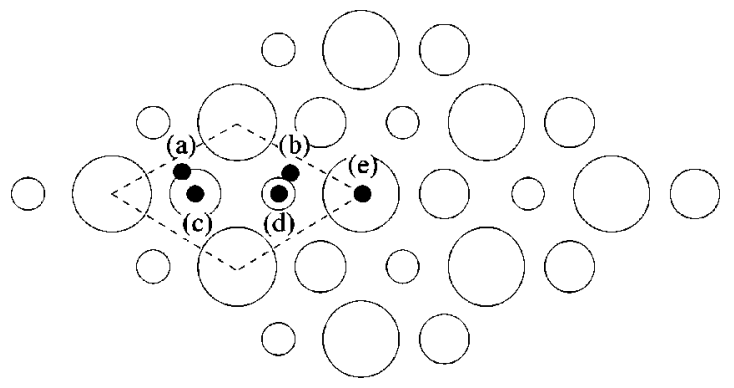

FIG. 1. Top view of the Au(111) surface. The large, middle, and small open circles denote the first layer, the second layer, and the third layer Au atoms, respectively. The closed circles denote the adsorption sites of the $\mathrm{S}$ atom of a benzenethiolate: (a) the hcp-bridge, (b) the fcc-bridge, (c) the hcp-hollow, (d) the fcc-hollow, and (e) the on-top sites. The dotted lines denote the $(1 \times 1)$ surface unit cell.

used for the exchange-correlation energy functional. ${ }^{15} \mathrm{We}$ have constructed ultrasoft pseudopotentials of $\mathrm{H} 1 s, \mathrm{C} 2 p$, and Au $5 d$ states by Vanderbilt's scheme ${ }^{16}$ and other states by norm-conserving scheme. The plane wave basis set with a cutoff energy of $25 \mathrm{Ry}$ is used for the expansion of wave functions. The $\mathrm{Au}(111)$ surface is modeled as a repeated slab geometry consisting of four layers of $\mathrm{Au}$ atoms and the vacuum region equivalent to six atomic layers. We have confirmed the convergence of the calculated adsorption energy within $0.05 \mathrm{eV}$ per one benzenethiolate molecule by changing these computational conditions. The $(\sqrt{3} \times 23)$ herringbone reconstruction of the bare $\mathrm{Au}(111)$ surface is reported to be lifted upon alkanethiolate adsorption by using the technique of $\mathrm{x}$-ray standing waves (XSWs). ${ }^{17}$ We assume in this study that the $\mathrm{Au}(111)$ surface forms the $(1 \times 1)$ structure initially. The adsorbed molecule and the top layer of substrate $\mathrm{Au}$ atoms are fully relaxed, while other substrate layers are fixed at the bulk positions.

For the adsorption geometry of a single molecule on the $\mathrm{Au}(111)$ surface, we use the $(3 \times 3)$ surface unit cell, which corresponds to a surface coverage of $\Theta=1 / 3$. Here we define full coverage $(\Theta=1)$ as one thiol molecule per three $\mathrm{Au}$ surface atoms. We use a uniform mesh of $36 k$-points for surface Brillouin zone sampling. By using larger surface unit cells, we have confirmed that the $(\sqrt{3} \times \sqrt{3})$ unit cell is sufficient to treat the single molecule adsorption in the low cov-
TABLE I. The adsorption energies and the structural parameters for the stable geometries of single benzenethiolate molecule adsorbed at the fccbridge, the hcp-bridge, the fcc-hollow, the hcp-hollow, and the on-top sites of the $\mathrm{Au}(111)$ surface. The tilting angle of benzenethiolate $(\theta)$ is defined as the angle of the $\mathrm{S}-\mathrm{C}$ bond with respect to the surface normal. The $r(\mathrm{~S}-\mathrm{Au})$ and $r(\mathrm{Au}-\mathrm{Au})$ are the distance between the adsorbed $\mathrm{S}$ atom and the nearest neighbor $\mathrm{Au}$ atoms and the longest distance between two surface $\mathrm{Au}$ atoms.

\begin{tabular}{lcccl}
\hline \hline \multicolumn{1}{c}{ Site } & $E_{\text {ad }}(\mathrm{eV})$ & $\theta\left(^{\circ}\right)$ & $r(\mathrm{~S}-\mathrm{Au})(\AA)$ & $r(\mathrm{Au}-\mathrm{Au})(\AA)$ \\
\hline fcc-bridge & $1.37(0)$ & 60.95 & 2.506 & $3.106(+5.8 \%)$ \\
hcp-bridge & $1.35(0.02)$ & 61.68 & 2.502 & $3.104(+5.8 \%)$ \\
fcc-hollow & $1.27(0.10)$ & 19.87 & 2.460 & $3.260(+11.1 \%)$ \\
hcp-hollow & $1.18(0.19)$ & 21.08 & 2.489 & $3.194(+8.8 \%)$ \\
On-top & $1.05(0.32)$ & 79.45 & 2.422 & $3.010(+2.6 \%)$ \\
\hline \hline
\end{tabular}

erage limit. The adsorption energy of the benzenethiolate molecule on the $\mathrm{Au}(111)$ surface is evaluated by

$$
\begin{aligned}
E_{\mathrm{ad}}= & -\left[E\left(\mathrm{C}_{6} \mathrm{H}_{5} \mathrm{~S} / \mathrm{Au}(111)\right)-(E(\mathrm{Au}(111))\right. \\
& \left.\left.+E\left(\mathrm{C}_{6} \mathrm{H}_{5} \mathrm{~S}\right)\right)\right],
\end{aligned}
$$

where $E\left(\mathrm{C}_{6} \mathrm{H}_{5} \mathrm{~S} / \mathrm{Au}(111)\right), E(\mathrm{Au}(111))$, and $E\left(\mathrm{C}_{6} \mathrm{H}_{5} \mathrm{~S}\right)$ are the total energies of the $\mathrm{C}_{6} \mathrm{H}_{5} \mathrm{~S}$ molecule adsorbed on the $\mathrm{Au}(111)$ surface, the clean $\mathrm{Au}(111)$, and the gas phase $\mathrm{C}_{6} \mathrm{H}_{5} \mathrm{~S}$ molecule, respectively. Along this definition, a positive value means an exothermic reaction.

For the adsorption geometry of SAM structures with $\Theta$ $=1$ on the $\mathrm{Au}(111)$ surface, we use the $(2 \sqrt{3} \times \sqrt{3})$ and the $(\sqrt{3} \times \sqrt{3})$ surface unit cells. For such high coverage, longrange van der Waals (vdW) interactions, or dispersion forces between benzenethiolates will play an important role in determining the adsorption geometry. However, the inclusion of the vdW interactions in density functional calculations is still under study and debate. In this study, we have empirically included the $\mathrm{vdW}$ interactions between molecules, by adding to the DFT energy functional a parameter-dependent term of the $-C / r^{6}$ form for the attractive $\mathrm{vdW}$ interaction tails. ${ }^{18,19}$ The coefficient $C$ between carbon atoms in benzene rings is set to be $-20.0 \AA^{6} \mathrm{eV}$. The details will be described elsewhere. ${ }^{20}$ (a)

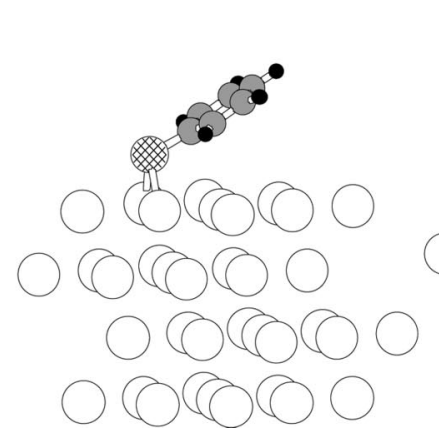

(b)

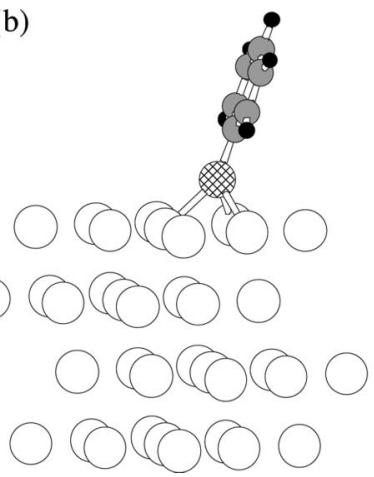

(c)

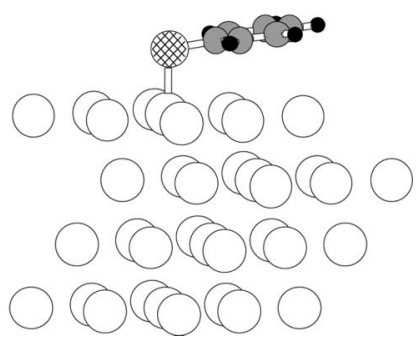

FIG. 2. Optimized atomic configurations for benzenethiolates adsorbed at (a) the fcc-bridge, (b) the fcc-hollow, and (c) the on-top sites of the Au(111) surface. The open, closed, gray, and hatched circles denote $\mathrm{Au}, \mathrm{H}, \mathrm{C}$, and $\mathrm{S}$ atoms, respectively. 
(a)

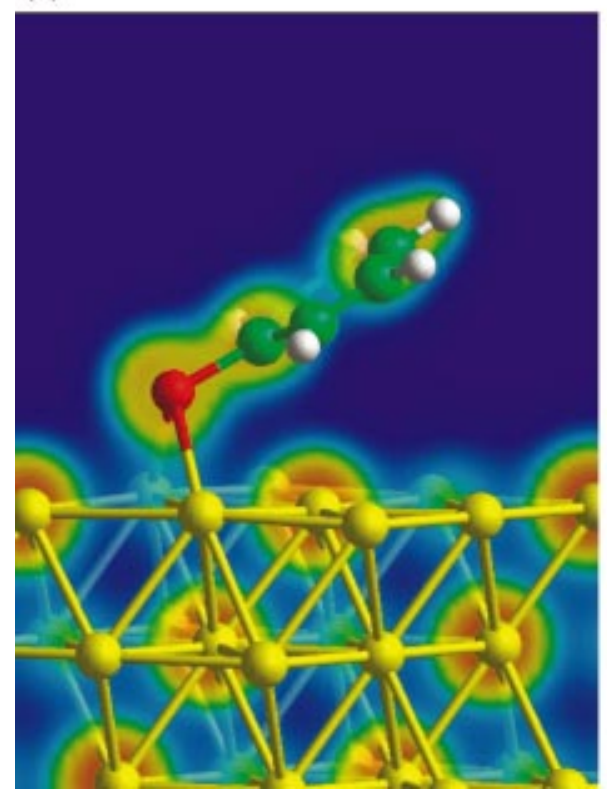

(b)

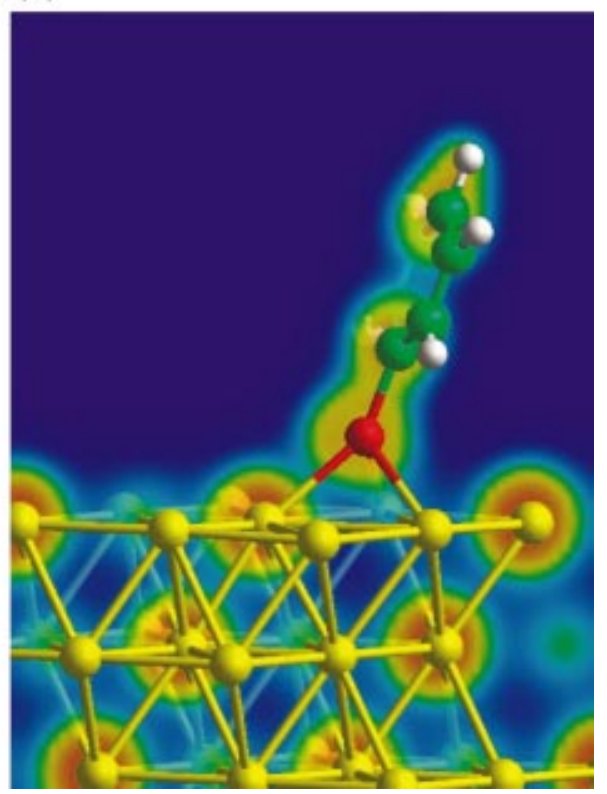

FIG. 3. (Color) Calculated charge density for benzenethiolates adsorbed at (a) the fcc-bridge and (b) the fcc-hollow sites of the Au(111) surface.

\section{RESULT AND DISCUSSION}

\section{A. Adsorption of benzenethiolate at low coverage}

First, we study the stable structures of the benzenethiolate adsorbed on the $\mathrm{Au}(111)$ surface by using the $(3 \times 3)$ surface unit cell, which would correspond to the low coverage limit. The adsorption geometry has a number of degrees of freedom, including the adsorption site of the $\mathrm{S}$ atom of the thiolate on the $\mathrm{Au}(111)$ surface (Fig. 1) and the tilting angle of the molecule $\theta$, which is defined as the angle of the S-C bond with respect to the surface normal. We have optimized the adsorption structures of the thiolate molecule, starting from various initial configurations.

Five stable adsorption structures are found for this system. The adsorption energies and the structural parameters of these stable adsorption geometries are summarized in Table I. In the most stable adsorption structure [Fig. 2(a)], the S atom of the thiolate is located at the bridge site slightly shifted to the fcc-hollow site (named the fcc-bridge site) and the molecule is tilted from the surface normal by $61^{\circ}$. The distances between the $\mathrm{S}$ atom and the two nearest neighbor $\mathrm{Au}$ atoms are $2.51 \AA$, whereas the distance between the $\mathrm{S}$ atom and the third nearest $\mathrm{Au}$ atom is $3.29 \AA$. The calculated charge density [Fig. 3(a)] clearly shows that there is no bonding between the $\mathrm{S}$ atom and the third nearest $\mathrm{Au}$ atom. Thus, the $\mathrm{S}$ atom is bonded to two $\mathrm{Au}$ atoms in the most stable geometry. The adsorption energy at the fcc-bridge site is calculated to be $1.37 \mathrm{eV}$. In the second most stable adsorption geometry, the $\mathrm{S}$ atom is positioned at the bridge site slightly shifted to the hcp-hollow site (named the hcp-bridge site) in turn. The atomic configuration is very similar to the most stable one. The third [Fig. 2(b)] and fourth stable structures are, respectively, the adsorption geometries of the $\mathrm{S}$ atom at the fcc-hollow site and the hcp-hollow site. In both geometries, the $\mathrm{S}$ atom is bonded to three $\mathrm{Au}$ atoms [Fig. 3(b)], and the thiolate molecule is tilted from the surface normal by about $20^{\circ}$. In the fifth stable adsorption structure [Fig. 2(c)], the $\mathrm{S}$ atom is located at the on-top site of the $\mathrm{Au}(111)$ surface, and the molecule is lying almost parallel to the surface with the tilting angle of $79^{\circ}$. The $\mathrm{S}$ atom forms a bond to one $\mathrm{Au}$ atom with the length of $2.42 \AA$, which is considerably smaller than the $\mathrm{S}-\mathrm{Au}$ bonds in other stable structures. In these stable geometries, the bond lengths within the thiolate molecule are almost the same as those in an isolate molecule except for the $\mathrm{S}-\mathrm{C}$ bond.

The adsorption of benzenethiolate relaxes substrate $\mathrm{Au}$ atoms considerably. Table II presents the energy gains induced by the Au relaxation, which are obtained from the energy differences between the benzenethiolate adsorbed on $\mathrm{Au}(111)$ with and without relaxation. The energy gain due to the Au relaxation is, for example, as large as $0.26 \mathrm{eV}$ for the adsorption at the fcc-hollow site, which is comparable to the energy differences between different adsorption geometries. This indicates that the Au relaxation is important in determining the stable adsorption geometry of the benzenethiolate on the $\mathrm{Au}(111)$ surface.

The adsorption site of the $\mathrm{S}$ atom is still in intense debate. Sellers et al. ${ }^{21}$ showed from cluster calculations that the most stable adsorption site of methylthiolate on $\mathrm{Au}(111)$ was the hollow site. Fenter et al. $^{17,22}$ suggested from XSW experiments that $\mathrm{S}$ atoms were adsorbed at two different adsorption sites such as the bridge and the hollow sites or the on-top and the hollow sites. Recently, Morikawa et al. ${ }^{12-14}$ concluded from DFT calculations using slab models that the

TABLE II. Energy gains due to the relaxation of surface Au atoms for benzenethiolates adsorbed at the fcc-bridge, the hcp-bridge, and the fcchollow sites of the $\mathrm{Au}(111)$ surface.

\begin{tabular}{cccc}
\hline \hline Site & fcc-bridge & hcp-bridge & fcc-hollow \\
\hline$\Delta E_{\mathrm{ad}}(\mathrm{eV})$ & 0.11 & 0.09 & 0.26 \\
\hline \hline
\end{tabular}




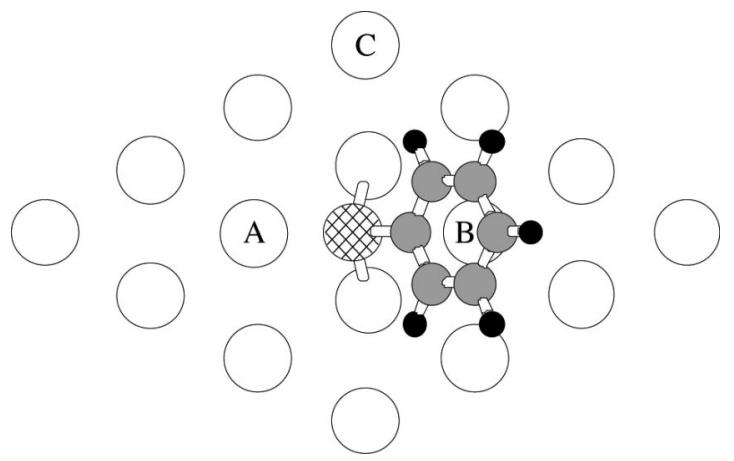

FIG. 4. Position of Au vacancy formed on the $\mathrm{Au}(111)$ surface with benzenethiolates adsorbed on it. Three positions (A, B, and C) are considered.

fcc-bridge site is the most stable. Other recent DFT calculations ${ }^{23-26}$ for alkanethiolate also supported the bridge configuration. The present result for benzenethiolate further confirms the bridge site, although benzenethiolate is a different type of thiolate. Very recently, however, Kondoh et al. ${ }^{27}$ claimed from $\mathrm{x}$-ray diffraction experiments that the most stable site of methylthiolate was the on-top site. None of the DFT calculations except for a very recent one by Bauschlicher and Ricca ${ }^{9}$ concluded the on-top site was the most stable. They claimed that the benzene- $(1,4)$-dithiolate was adsorbed at the on-top site from quantum chemical calculations using a cluster of $25 \mathrm{Au}$ atoms. In their calculations, however, an insufficient basis set was used. Only five Au atoms in the cluster were described by a large basis set with $d$ electrons, others by a small basis set with no $d$ electrons, and furthermore the relaxation of the surface Au atoms was neglected.

As shown in Table $\mathrm{I}$, the $\mathrm{Au}-\mathrm{Au}$ distances around the $\mathrm{S}$ atom adsorbed on the $\mathrm{Au}(111)$ surface are extended by several percent compared with the bulk value. This means that the surface Au atoms repel with each other. This repulsive $\mathrm{Au}-\mathrm{Au}$ interaction tends to facilitate the vacancy formation on the $\mathrm{Au}(111)$ surface, which has been suggested recently. ${ }^{14,28}$ We examine the possibility of the vacancy formation for the most stable adsorption geometry of benzenethiolates, that is, the adsorption at the fcc-bridge site. One Au vacancy is formed at three distinct positions $(A, B$, and $C$ ) around the $\mathrm{S}$ atom, as shown in Fig. 4. The larger $(4 \times 4)$ unit cell is used for the vacancy formation in order to allow a sufficient surface relaxation around the Au vacancy. The vacancy formation energy is evaluated by

$$
E_{\mathrm{vac}}=E(\text { one vacancy })+\mu_{\mathrm{Au}}(\text { bulk })-E(\text { no vacancy }),
$$

where $E$ (one vacancy), $E$ (no vacancy), and $\mu_{\mathrm{Au}}$ (bulk) are the total energies of the adsorption structures with one $\mathrm{Au}$ vacancy and without vacancy, and a Au atom in the bulk, respectively. Along this definition, a positive value means an endothermic reaction. The obtained vacancy formation energies are $0.28,0.40$, and $0.60 \mathrm{eV}$ for the $A, B$, and $C$ sites, respectively, which are smaller than that of $0.64 \mathrm{eV}$ on the clean $\mathrm{Au}(111)$ surface. The adsorption of benzenethiolates makes the vacancy formation easier on the $\mathrm{Au}(111)$ surface, although it is still endothermic.

\section{B. Adsorption process}

There are some experimental reports on the adsorption of benzenethiol on metal surfaces from the gas phase. ${ }^{29,30} \mathrm{We}$ investigate the dissociative adsorption process of benzenethiol molecules on the $\mathrm{Au}(111)$ surface. As shown in Fig. 5, we assume the overall adsorption process as follows: (a) Benzenethiol molecules in the vacuum approach the $\mathrm{Au}(111)$ surface and (b) adsorb on it in a physisorbed state. (c) Following the physisorption, the benzenethiol molecules are dissociated into thiolate molecules and $\mathrm{H}$ atoms, both of which are adsorbed on the surface. (d) The adsorbed $\mathrm{H}$ atoms are desorbed in the gas phase by forming hydrogen molecules. The total energy of the benzenethiol molecule in the vacuum and the $\mathrm{Au}(111)$ surface [Fig. 5(a)] is taken as a reference energy of the adsorption process. The physisorption energy of the benzenethiol molecule on the $\mathrm{Au}(111)$ surface [Fig. 5(b)] is calculated to be $0.07 \mathrm{eV}$, which is quite small compared with that of $0.32 \mathrm{eV}$ in the case of the alkanethiol physisorption. ${ }^{26}$ The energy of the dissociative adsorption geometry [Fig. 5(c)] is $0.22 \mathrm{eV}$, which means that the dissociation of the benzenthiol molecule is energetically unfavorable. On the other hand, the formation of hydrogen molecules in the gas phase from $\mathrm{H}$ atoms adsorbed on the surface is exothermic by $0.42 \mathrm{eV}$ per hydrogen atom. As a result, the dissociative adsorption of benzenethiol molecules on the $\mathrm{Au}(111)$ surface with the desorption of hydrogen molecules from the surface being involved is energetically favorable by $0.20 \mathrm{eV}$. Although we have not investigated the ac-

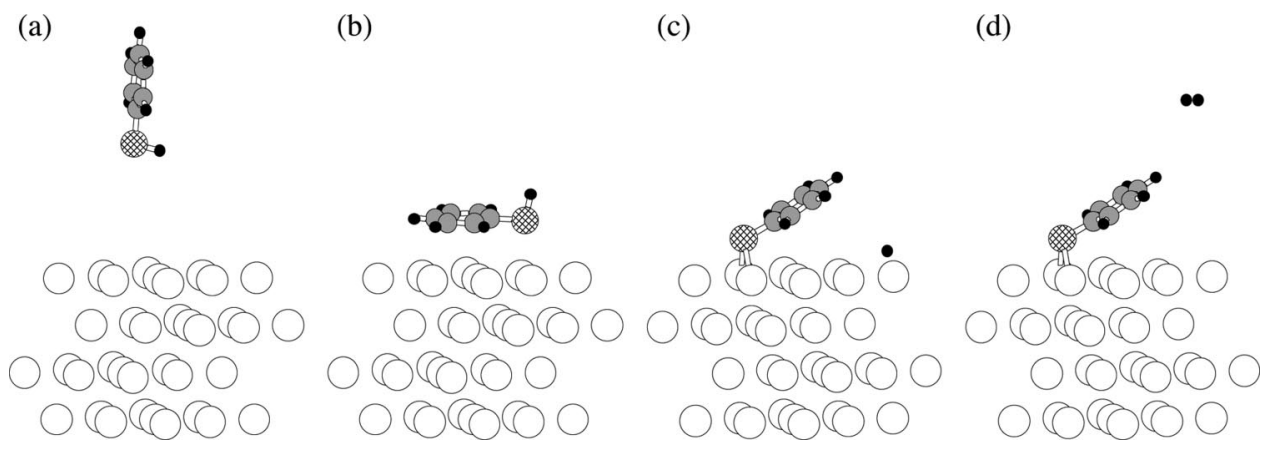

FIG. 5. Overall process of dissociative adsorption of benzenethiol molecules on the Au(111) surface from the gas phase: (a) benzenethiol molecules in the gas phase, (b) physisorption of molecules, (c) dissociative adsorption into thiolates and $\mathrm{H}$ atoms, and (d) desorption of hydrogen molecules. 
(a)

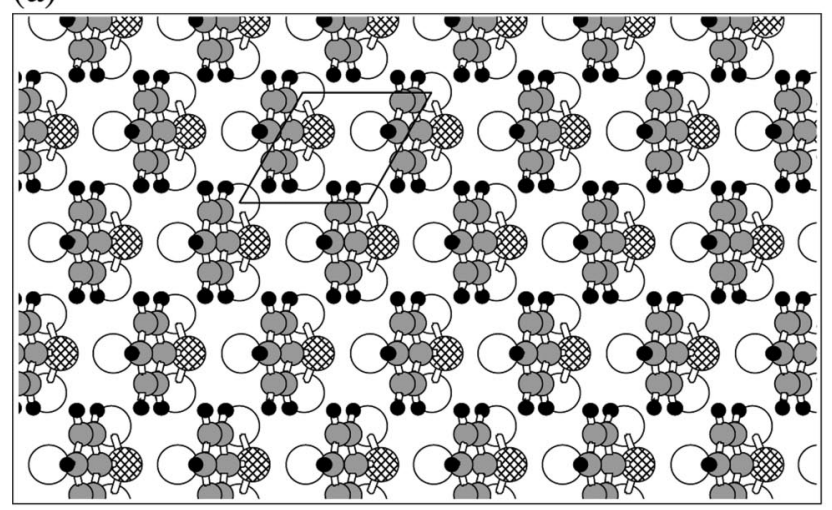

(b)

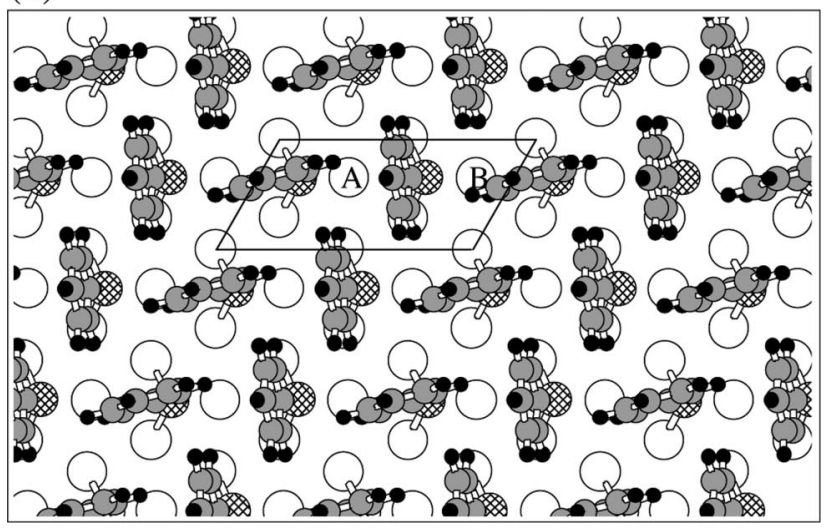

FIG. 6. Optimized atomic configurations for (a) $(\sqrt{3} \times \sqrt{3})$ and (b) $(2 \sqrt{3}$ $\times \sqrt{3})$ SAM structures of benzenethiolates adsorbed at the bridge sites of the $\mathrm{Au}(111)$ surface. Positions of Au vacancy under consideration (A, B) are also shown in the $(2 \sqrt{3} \times \sqrt{3})$ structure.

tivation barrier for the dissociative adsorption process, the obtained results indicate that the formation of benzenethiol SAMs on the $\mathrm{Au}(111)$ surface from the gas phase is energetically preferable at least.

\section{Adsorption at high coverage}

Next, we examine the SAM structures of benzenethiol molecules on the $\mathrm{Au}(111)$ surface. Dhirani et al. investigated the structures of monolayers of oligo(phenylethynyl) benzenethiols on $\mathrm{Au}(111)$ and revealed that the degree of order in these systems increases with chain length. They reported that phenylethynyl benzenethiol having three benzene rings forms a well-ordered SAM structure with $(2 \sqrt{3} \times \sqrt{3})$ surface periodicity that is named the "herringbone" structure, whereas benzenethiol with one benzene ring does not form an ordered phase. ${ }^{10}$ The $(2 \sqrt{3} \times \sqrt{3})$ herringbone surface comprises two crystallographically distinct benzenethiolate molecules. It is suggested that the distinct molecular features come from different orientations of the benzene rings, as shown in Fig. 6(b). On the other hand, Wan et al. reported that benzenethiol forms a well-ordered $(\sqrt{13} \times \sqrt{13})$ monolayer structure. ${ }^{11}$ It is still unclear whether benzenethiol forms any ordered phase on the $\mathrm{Au}(111)$ surface. We investigate the SAM structures with $(2 \sqrt{3} \times \sqrt{3})$ periodicity, as well as the structures with $(\sqrt{3} \times \sqrt{3})$ periodicity for comparison, both of which correspond to the full coverage $(\Theta$ $=1$ ). Table III summarizes the results for the formation energies of the SAM structures, which are evaluated from isolated benzenethiolate molecules. In these calculations, longrange van der Waals interactions between benzene rings are included as described earlier.

For the SAM structures with the $(\sqrt{3} \times \sqrt{3})$ periodicity, we consider three adsorption sites: the bridge, the fcc-hollow, and the on-top sites. In the optimized $(\sqrt{3} \times \sqrt{3})$ SAM structures, the benzenthiolates are oriented closer to the upright position compared with the case of the single molecule adsorption. This is due to the interaction between molecules. In the SAM geometry adsorbed at the bridge site [Fig. 6(a)], the benzenthiolate is tilted from the surface normal by $33^{\circ}$, which is indeed much smaller than the value of $61^{\circ}$ for the single molecule adsorption at this site [Fig. 2(a)]. At the fcc-hollow and the on-top sites the molecules are almost standing upright. The SAM structures at the bridge and the fcc-hollow sites are almost degenerate, whereas the on-top site is much higher in energy. This is because the upright orientation is significantly unstable for the molecule adsorbed at the on-top site.

We also consider the $(2 \sqrt{3} \times \sqrt{3})$ SAM structures adsorbed at the bridge and the fcc-hollow sites. Both $(2 \sqrt{3}$ $\times \sqrt{3})$ structures are found to be more stable than the $(\sqrt{3}$ $\times \sqrt{3}$ ) counterparts. The most stable geometry among the SAM structures under consideration is the $(2 \sqrt{3} \times \sqrt{3})$ structure adsorbed at the bridge site, which is consistent with the herringbone structures observed for phenylethynyl benzenethiol having three benzene rings. ${ }^{10}$ In the optimized geometry [Fig. 6(b)], the molecule is tilted from the surface normal by $21^{\circ}$. This result is close to the tilted orientations of $14^{\circ 6}$ and $30^{\circ 11}$ determined experimentally, but largely different from the reported flat orientation of $76^{\circ}$.

TABLE III. Formation energies, $E_{f}$, of $(2 \sqrt{3} \times \sqrt{3})$ and $(\sqrt{3} \times \sqrt{3})$ SAM structures adsorbed on the $\mathrm{Au}(111)$ surface. Two contributions to the formation energy are given, the interaction between the molecules $(\mathrm{M}-\mathrm{M})$ and the interaction between the molecules and the $\mathrm{Au}(111)$ surface $(\mathrm{M}-\mathrm{S})$. The interaction between the molecules is divided into the contributions from the DFT energy functional (DFT) and from the vdW interactions (vdW).

\begin{tabular}{|c|c|c|c|c|c|}
\hline \multirow{2}{*}{$\begin{array}{l}\text { Periodicity } \\
\text { site }\end{array}$} & \multicolumn{2}{|c|}{$(2 \sqrt{3} \times \sqrt{3}) R 30^{\circ}$} & \multicolumn{3}{|c|}{$(\sqrt{3} \times \sqrt{3}) R 30^{\circ}$} \\
\hline & Bridge & fcc-hollow & Bridge & fcc-hollow & on-top \\
\hline$E_{f}(\mathrm{eV})$ & 1.37 & 1.32 & 1.18 & 1.19 & 0.30 \\
\hline $\mathrm{M}-\mathrm{M}(\mathrm{eV})$ Total & 0.10 & 0.11 & -0.10 & -0.03 & -0.03 \\
\hline DFT & -0.10 & -0.09 & -0.35 & -0.26 & -0.26 \\
\hline $\mathrm{vdW}$ & 0.20 & 0.20 & 0.25 & 0.23 & 0.23 \\
\hline $\mathrm{M}-\mathrm{S}(\mathrm{eV})$ & 1.27 & 1.21 & 1.28 & 1.22 & 0.33 \\
\hline
\end{tabular}


There are two contributions to the formation energy of the SAM structure on the $\mathrm{Au}(111)$ surface: the interaction between the molecules and that between the molecules and the $\mathrm{Au}(111)$ surface, which are given in Table III. The interaction between the molecules can be evaluated from the formation energy of the free-standing SAM structure apart from the $\mathrm{Au}(111)$ surface, and can be divided into the contributions from the DFT energy functional and from the vdW interactions. The molecule-surface interaction is given by the binding energy of the free-standing SAM to the $\mathrm{Au}(111)$ surface. Here we make a comparison between the $(2 \sqrt{3}$ $\times \sqrt{3})$ and the $(\sqrt{3} \times \sqrt{3})$ SAM structures adsorbed at the bridge site. As for the molecule-surface interaction, both SAM structures are slightly different. The binding energies of the free-standing SAM to the Au(111) surface are weaker than the adsorption energy of isolated molecules, since the molecules in the SAM structure are hindered from relaxing to the most stable $\mathrm{Au}-\mathrm{S}$ bonding geometry. The moleculemolecule interaction causes a difference between the two SAM structures. The formation energy for the free-standing $(\sqrt{3} \times \sqrt{3})$ SAM structure is found to be negative $(-0.10 \mathrm{eV})$. This means that there are repulsive interactions between thiolate molecules due to steric hindrance, which arise from the DFT contribution $(-0.35 \mathrm{eV})$ to the formation energy. In the $(2 \sqrt{3} \times \sqrt{3})$ structure, the repulsive DFT contribution is much reduced by $0.25 \mathrm{eV}$ compared with the $(\sqrt{3} \times \sqrt{3})$ structure, which indicates that the $(2 \sqrt{3} \times \sqrt{3})$ structure is to a great extent sterically relaxed. Since the attractive vdW contribution $(0.20 \mathrm{eV})$ dominates the repulsive DFT one $(-0.10 \mathrm{eV})$, the formation of the free-standing $(2 \sqrt{3} \times \sqrt{3})$ SAM structure is found to be energetically favorable. In this way, the $(2 \sqrt{3} \times \sqrt{3})$ SAM structure results from the interactions between molecules.

For the $(2 \sqrt{3} \times \sqrt{3})$ SAM structure adsorbed at the bridge site on the $\mathrm{Au}(111)$ surface, we examine the possibility of the vacancy formation, where one Au vacancy is formed at two distinct positions $(A$ and $B)$ in the $(2 \sqrt{3} \times \sqrt{3})$ unit cell [Fig. $6(b)]$. The vacancy formation for both sites is found to be exothermic with the formation energy of $-0.24 \mathrm{eV}$. The high coverage of thiolates $(\Theta=1)$ induces a stronger $\mathrm{Au}-\mathrm{Au}$ repulsion in the SAM structure, which makes the vacancy formation exothermic, different from the single adsorption case of $\Theta=1 / 3$. $^{31}$ In the optimized SAM structure with one $\mathrm{Au}$ vacancy, the heights of the two benzenthiolates in the $(2 \sqrt{3}$ $\times \sqrt{3}$ ) surface unit cell are different. This is because the vacancy formation induces a difference in the tilting angle between the two molecules, which leads to a slight corrugation in the molecular height. The outermost $\mathrm{C}$ atom of one molecule is indeed located $0.2 \AA$ above the outermost $\mathrm{C}$ atom of the other, which is quite in contrast with a negligible height difference of $0.001 \AA$ without vacancy. Figure 7 presents the simulated STM image for the $(2 \sqrt{3} \times \sqrt{3})$ SAM structure with vacancy, which is the charge density integrated from the valence bands covering a range of $1 \mathrm{eV}$ above the Fermi level. The simulated scanning tunneling microscopy (STM) image is qualitatively consistent with experimentally observed STM images, ${ }^{10}$ in which one molecule in the $(2 \sqrt{3}$ $\times \sqrt{3}$ ) unit cell is imaged brighter than the other, although

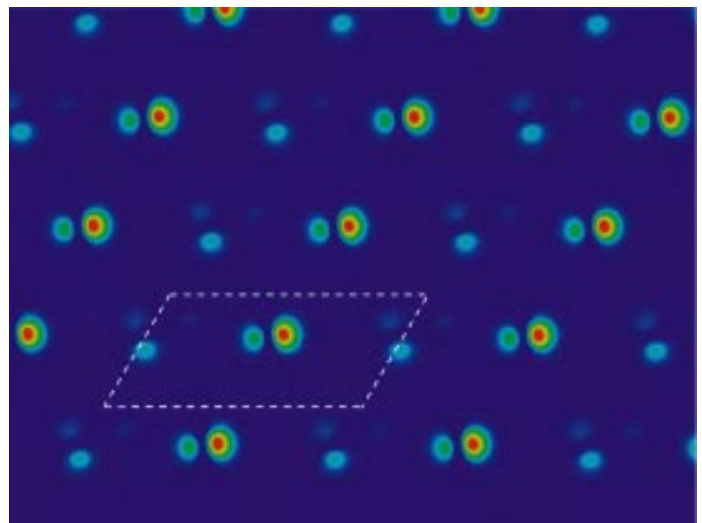

FIG. 7. (Color) Surface charge density calculated for the $(2 \sqrt{3} \times \sqrt{3})$ SAM structure of benzenethiolates adsorbed at the bridge sites of the $\mathrm{Au}(111)$ surface with $\mathrm{Au}$ vacancies at the A site. The local charge density is integrated from the valence bands covering $1 \mathrm{eV}$ above the Fermi level, which is cut at $0.56 \AA$ above the outermost $\mathrm{C}$ atom. The dotted lines denote the $(2 \sqrt{3} \times \sqrt{3}) \mathrm{R} 30^{\circ}$ surface unit cell.

the numbers of benzene rings in the molecules are different between the present study and the experimental work.

\section{CONCLUSION}

In summary, we have investigated the adsorption of benzenethiolate on the $\mathrm{Au}(111)$ surface by using the firstprinciples calculations. For the adsorption of a single molecule, the benzenethiolate is adsorbed at the bridge site slightly shifted toward the fcc-hollow site, and is tilted by $61^{\circ}$ from the surface normal. For the SAM structures, the $(2 \sqrt{3} \times \sqrt{3})$ herringbone structure is stabilized against the $(\sqrt{3} \times \sqrt{3})$ structure by large steric relaxation. In the most stable $(2 \sqrt{3} \times \sqrt{3})$ SAM structure, the molecule is adsorbed at the bridge site and oriented much closer to the upright position compared with the case of the single molecule adsorption. The van der Waals interaction plays an important role in forming the SAM structure. The adsorption of benzenethiolates induces the repulsive $\mathrm{Au}-\mathrm{Au}$ interaction, which facilitates the formation of surface Au vacancy. This is the first detailed theoretical analysis of the adsorption geometry of aromatic thiol molecules on the $\mathrm{Au}(111)$ surface. Our results provide important knowledge for understanding the electronic properties of aromatic molecules adsorbed on metallic substrates.

\section{ACKNOWLEDGMENTS}

This research is partially supported by ACT-JST, and also by FSIS and Special Coordination Funds of MEXT of the Japanese Government. Y.M. acknowledges NISSAN SCIENCE FOUNDATION. The present calculations were carried out using the Numerical Materials Simulator in NIMS.

${ }^{1}$ G. E. Poirier, Chem. Rev. 97, 1117 (1997).

${ }^{2}$ A. Ulman, Chem. Rev. 96, 1533 (1996).

${ }^{3}$ F. Schreiber, Prog. Surf. Sci. 65, 151 (2000).

${ }^{4}$ L. A. Bumm, J. J. Arnold, M. T. Cygan, T. D. Dunbar, T. P. Burgin, L. Jones II, D. L. Allara, J. M. Tour, and P. S. Weiss, Science 271, 1705 (1996). 
${ }^{5}$ M. A. Reed, C. Zhou, C. J. Muller, T. P. Burgin, and J. M. Tour, Science 278, 252 (1997).

${ }^{6}$ K. T. Carron and L. G. Hurley, J. Phys. Chem. 95, 9979 (1991).

${ }^{7}$ C. A. Szafranski, W. Tanner, P. E. Laibinis, and R. L. Garrell, Langmuir 14, 3570 (1998).

${ }^{8}$ A. Johansson and S. Stafstroem, Chem. Phys. Lett. 322, 301 (2000).

${ }^{9}$ C. W. Bauschlicher Jr. and A. Ricca, Chem. Phys. Lett. 367, 90 (2003).

${ }^{10}$ A.-A. Dhirani, R. W. Zehner, R. P. Hsung, P. Guyot-Sionnest, and L. R. Sita, J. Am. Chem. Soc. 118, 3319 (1996).

${ }^{11}$ L.-J. Wan, M. Terashima, H. Noda, and M. Osawa, J. Phys. Chem. B 104, 3563 (2000).

${ }^{12}$ T. Hayashi, Y. Morikawa, and H. Nozoye, J. Chem. Phys. 114, 7615 (2001).

${ }^{13}$ Y. Morikawa, T. Hayashi, C. C. Liew, and H. Nozoye, Surf. Sci. 507-510, 46 (2002).

${ }^{14}$ Y. Morikawa, C. C. Liew, and H. Nozoye, Surf. Sci. 514, 389 (2002).

${ }^{15}$ J. P. Perdew, K. Burke, and M. Ernzerhof, Phys. Rev. Lett. 77, 3865 (1996).

${ }^{16}$ D. Vanderbilt, Phys. Rev. B 41, 7892 (1990).

${ }^{17}$ P. Fenter, F. Schreiber, L. Berman, G. Scoles, P. Eisenberger, and M. J. Bedzzyk, Surf. Sci. 412/413, 213 (1998).

${ }^{18}$ S. Serra, S. Iarlori, E. Tosatti, S. Scandolo, and G. Santoro, Chem. Phys. Lett. 331, 339 (2000).
${ }^{19}$ S. Tsuzuki and H. P. Luethi, J. Chem. Phys. 114, 3949 (2001); S. Tsuzuki, K. Honda, T. Uchimaru, M. Mikami, and K. Tanabe, J. Am. Chem. Soc. 124, 104 (2002).

${ }^{20}$ Y. Morikawa, S. Tsuzuki, and C. C. Liew (unpublished).

${ }^{21}$ H. Sellers, A. Ulman, Y. Shnidman, and J. E. Eilers, J. Am. Chem. Soc. 115, 9389 (1993).

${ }^{22}$ P. Fenter, A. Eberhard, and P. Eisenberger, Science 266, 1216 (1994).

${ }^{23}$ M. C. Vargas, P. Giannozzi, A. Selloni, and G. Scoles, J. Phys. Chem. B 105, 9509 (2001).

${ }^{24}$ Y. Akinaga, T. Nakajima, and K. Hirao, J. Chem. Phys. 114, 8555 (2001).

${ }^{25}$ J. Gottschalck and B. J. Hammer, J. Chem. Phys. 116, 784 (2002).

${ }^{26}$ Y. Yourdshahyan and A. M. Rappe, J. Chem. Phys. 117, 825 (2002).

${ }^{27}$ H. Kondoh, M. Iwasaki, T. Shimada, K. Amemiya, T. Yokoyama, T. Ohta, M. Shimomura, and S. Kono, Phys. Rev. Lett. 90, 066102 (2003).

${ }^{28}$ L. M. Molina and B. Hammer, Chem. Phys. Lett. 360, 264 (2002).

${ }^{29}$ D. M. Jaffey and R. J. Madix, J. Am. Chem. Soc. 116, 3020 (1994).

${ }^{30}$ Y. Takata, T. Yokoyama, S. Yagi, N. Happo, H. Sato, K. Seki, T. Ohta, Y. Kitajima, and H. Kuroda, Surf. Sci. 259, 266 (1991).

${ }^{31}$ It is noted that the present GGA calculation tends to overestimate the vacancy formation energy, whereas the opposite is true with the LDA calculation. See Ref. 14 